\title{
Molecular Diagnosis and Palliative Care
}

\author{
Imane Abdellaoui Maane, Hicham El Hadi, Hassan Sefrioui, and Abdeladim \\ Moumen* \\ MASCIR: Moroccan Foundation for Advanced Science, Innovation and Research Rabat, 11 100, Morocco
}

Received: December 24, 2013; Accepted: February 07, 2014; Published: February 10, 2014

*Corresponding author: Abdeladim Moumen, MSc, Ph.D, HDR. MAScIR: Moroccan Foundation for Advanced Sciences, Innovation and Research MASCIR Medical Biotechnology Center MAScIR, Rabat Design, Rue Mohamed El Jazouli, Madinat Al Irfane 10100 Rabat, Morocco, Tel: 212-622702062; Fax : 212530 410501; E-mail: a.moumen@mascir.com

\begin{abstract}
Being diagnosed with a dangerous disease such as cancer is scary and turns the life upside down. Patients affected will be faced with the need to make health care decisions, plan for their future and their family's one. Everything feels overwhelming with a sufferance from pain and other unpleasant symptoms. Palliative care is a specialized medical care focused on relief of the pain, symptoms and stress of serious illness. Its main aim is to improve quality of life for both patients and their family. It is appropriate at any age and at any stage in your illness and can be provided along with treatment meant to cure. Diagnostic tests are prerequisite to a strong health care system. In this review we aim to shed light on the role played by molecular diagnosis in the improvement of palliative care system.
\end{abstract}

\section{Introduction}

Death and dying are not easy to deal with. But there is a way to ease pain and make life better for people who are dying and for their loved ones [1]. Palliative care is an approach that improves the quality of life of patients and their families facing the problem associated with life-threatening illness, through the prevention and relief of suffering by means of early identification and impeccable assessment and treatment of pain and other problems, physical, psychosocial and spiritual [2]. It is noteworthy that palliative care aims to provide the best quality of life for both adults and children living with and affected by lifelimiting illness.

Once the diagnosis, palliative care are applicable in the course of the illness until death and are performed according to a draft of continuing care that emphasizes listening, communication, establishing confidence with the patient and his family [3]. Indeed, depending on the stage, priority will be given to both support for physical comfort, emotional and moral patient and mainly clinical investigations and treatments to cure or decelerate the progression of the disease in conjunction with other therapies that are intended to prolong life such as chemotherapy \& radiotherapy [4]. The relative importance of these two paths is regularly reassessed and implementation of palliative care should be a consensus among the healthcare team, the patient and his relatives if the patient wishes. Diagnosis is undoubtedly an important step toward accurate clinical investigation and treatment orientation. It provides a substantial amount of information that could allow better management of the palliative care system [5]. Due to the lack in the literature of supporting data that correlate palliative care and molecular diagnostics, this review could constitute a kind of starting point that could trigger discussion on this very interesting point.

\section{Diagnosis Evolution}

Diagnostic tests are the support of a strong health care system, they play a decisive role in making decision and help to direct patients to appropriate care, modifying their treatment and accompany them with general or specialist supportive care [5]. By quantitative measurements diagnosis provides information on the stages of prevention, detection, care and treatment as well as effective management of health problems [6]. The adoption of molecular diagnostics has revolutionized health care as new ideas in research and treatment of many diseases have emerged. Indeed, molecular diagnostics allows detection and measurement of genetic material or proteins characteristic of a specific health condition or pathology. This diagnosis can explore the underlying mechanisms of the disease and thus establish personalized care. Continuous innovation in the molecular biology area has led to an increase of both the performance and speed of molecular diagnosis. Furthermore, the automation of techniques makes it reachable in health care settings. Molecular diagnosis is currently based on a number of techniques to detect and quantify nucleic acids and specific proteins. Indeed, while methods of genetic testing such as in situ hybridization or detection tests such as protein mass spectrometry are widely used in the molecular diagnostic area, polymerase chain reaction remains one of the most fundamental techniques used to amplify specific sequences of DNA or RNA in molecular diagnostic tests [7].

\section{Polymerase Chain Reaction (PCR) and its evolving influence on the health system}

The molecular diagnostic methods have revolutionized medical diagnosis and have become indispensable for routine analysis in the laboratory. In many areas of infectious diseases, conventional methods of detection have been replaced or 
supplemented by PCR tests. As a result, more diseases are detected early and the chances of success of treatment applied are great. Molecular tests enable rapid and specific diagnosis by direct detection of the genetic materials (DNA or RNA) of either an infectious factor or defective cells in order to implement a specific therapeutic treatment to the patient or even a prognosis. PCR, as a well established molecular test, represents an advanced technique that can detect short fragments of genes or specific mutations associated with physiological dysfunction or pathology. PCR detects and amplifies a portion of DNA from a consensus sequence by using the same enzymatic process utilized during the natural DNA replication. With its system of amplification, PCR allows detection of tiny amount of specific DNA segments present on a given sample [8].

The detection of DNA amplified by PCR has highly evolved, it went from gel electrophoresis by applying an electric current to the use of fluorescent dyes that specifically binds to the DNA fragment of interest, and the latter has led to the so called quantitative real-time PCR. Automated instruments have been developed to monitor the fluorescence generated during the amplification process. This allowed both the detection of the target DNA, and its quantification. This technique could be either used to determine the prevalence of an infectious agent in the body or to identify various genetic defects within a given cell sample. The use of PCR for the detection of many diseases evolved rapidly in the last years. From the detection of a mutation in a single gene test, up to the quantification of viral load to finally get to test multiplexing targeting multiple sequences at once [9].

Most PCR tests use chemical reactions that are based on rapid cycles of temperature to synthesize new DNA strands. The amplified DNA can be analyzed in multiple ways depending on the desired result. In automated real-time PCR systems, the use of dyes or other fluorescent chemicals that could be detected by specialized instruments allows detection of DNA amplification as it occurs, without any intervention of a laboratory staff. The results are generated as amplification curves that could be used for an accurate quantification of the amplified DNA. Intensive research efforts are continuing to simplify and decrease the time for the PCR process. Indeed, a recent isothermal method that does not rely on the temperature variation at each cycle is an example of a simplified PCR testing. This method could be performed outside the laboratory with only basic equipments such as small kettles and therefore within health quite basic care settings $[10,11]$.

\section{Palliative care in nonmalignant and malignant pathologies}

Around the world thousands of people are diagnosed with dangerous and deadly diseases such as cancer or HIV, which causes a considerable impact both economically and psychologically for the patient and his family [12]. The only alternative that can meet the needs of patients and improve their quality of life is the development of an effective and inexpensive approach to palliative care [13].

\section{Effective approaches to palliative care in malignant pathologies}

The support of patients with cancer through treatment may well involve input from a wide range of surgical, medical, allied medicine health professionals and laboratory disciplines. Indeed, a preliminary diagnosis is established by a physician or surgeon and subsequently confirmed by biopsy or fine-needle aspiration. Therefore an oncologist may undertake definitive treatment $[14,15]$.

But in most countries of the world, a large majority of patients coming for a first consultation are diagnosed at advanced stages. So the only realistic outcome is relief pain and palliative care. The palliative treatment is indicated when curative treatment is no longer possible, so the treatment may have anticancer activity acting on symptoms related to cancer [7]. In a lot of cases the improvement of symptoms was accompanied by a regression of the tumor mass observed clinically or by radiological techniques such as IRM. In other cases there may be a palliative improvement without regression of the tumor [16].

\section{Prostate cancer}

The prostate cancer represents $30 \%$ of cancers diagnosed in men, and after the lung cancer it constitutes the second leading cause of death from cancer in men over 60 years. In recent years the incidence of this cancer increased significantly, probably due to the molecular screening activity carried out by serum PSA (prostate specific antigen) and digital rectal examination [17].

However, many prostate tumors are clinically insignificant and will not cause symptoms in the patient's lifetime and will never evolve [18]. However, it is important to recognize those patients whose tumors are likely to become clinically apparent. This raises the importance of molecular diagnostics, targeting more specific markers that could provide more information about the likely progression to metastatic $[19,20]$. The treatment of prostate cancer differs according to the general condition of the patient and the stage of the disease. A radical local treatment can be considered as prostatectomy or radiation therapy, or hormonal therapy administered especially when metastases occur. The natural history of prostate cancer is most variable, usually in patients with bone metastases life expectancy is 5 years after diagnosis. The majority of tumors are sensitive to androgens. But the prognosis for patients whose disease has progressed through anti-androgen therapy is poor [21]. However these hormone treatments could involve many unpleasant sideeffects such as loss of libido and potency, hot flushes, change in fat deposition, osteoporosis, poor concentration and decreased energy all of which require palliative care for the patients [22]. It is indeed that palliative care is more relevant for more advanced stages of prostate cancer. Nevertheless, in our point of view, early detection and identification of prostate cancer using molecular tools could be very useful as a preparatory way of a more efficient palliative system.

\section{In nonmalignant pathologies}

Many molecular diagnostics improve monitoring and 
management of disease. Some of the most common monitoring molecular tests are tests used for the measurement of the HIV viral load in patients affected with HIV. Monitoring the viral load is achieved by amplification test that measure the amount of viral particles in order to determine the copy number of the virus present in the blood. It is necessary to maintain levels of viral load lower than possible to live with the disease. The viral load tests are not only used for diagnosis, but also to monitor disease progression and assess response to different antiretroviral treatment. Indeed periodic monitoring of viral load indicates whether the disease progresses, if the patient responds to treatment or if treatment is no longer working [23].

The natural progression of infection by HIV is very variable. For many patients there are few symptoms initially and it is only after several years of relatively normal health that the typical clinical features of AIDS become apparent. In others, the initial seroconversion may be more pronounced with fever. But the prognosis for patients whose disease has progressed through anti-retroviral therapy is poor [24]. These symptoms usually resolve spontaneously over a few weeks. While a small proportion of infected people may progress rapidly to full-blown AIDS within one or two years, in the majority AIDS takes eight to ten years to develop. A few individuals progress very slowly and may be still alive after 20 years, even without ARVs. As the immune system begins to become exhausted, the person becomes susceptible to a wide range of infections including recurrent upper respiratory tract infections, pneumonia, tuberculosis, a wide variety of intestinal micro-organisms, cryptococcal meningitis, herpes zoster and monilial infections of the mouth, vagina and esophagus $[25,26]$.

Clinical practice of palliatives care of HIV patients depends primarily of a decision making system based on current tangible evidence. Molecular diagnosis may give better understanding to the course of the pathology and give best monitoring of this category of patients for which psychological supports should accompany them from the first stages. The overall reporting rate for HIV/AIDS articles in palliative care journals is very low and there were no randomized clinical trials and systematic reviews found. In this context, a reporting of HIV/AIDS- A Systematic Review and Quantitative Analysis of Research Publications in Palliative Care Journals indicate a lack of adequate evidence base for HIV/AIDS in palliative care literature, and there is no information about molecular diagnosis application related to palliatives care [27].

The variability of the resilience of the immune system and the potentially fatal nature of some of the infections, make it difficult to estimate the prognosis of an individual. Anti-retroviral drugs (ARVs) do not cure AIDS, but merely delay its natural progress [28]. For HIV patients, died with AIDS is inevitable. Apart from a complex disease with prolonged suffering, emotional social and economic impacts are also heavy. Fear, stigma, rejection, grief make the disease is hard to bear. All of these facts make importance of palliative care for these patients [29].

\section{Conclusion}

Molecular diagnostics are continuously expanding and their use in biomedical laboratories is becoming a routine. The importance of molecular diagnostics in the rapid detection and identification as well as follow up of many threatening illnesses has made them a requirement for a solid health system in general and in particular palliative care system. Palliative care can be regarded as an approach which supports, in the best way possible, people with life-threatening illness and those close to them. It should be a right not a privilege. This means putting patients at the center of care, empowering them, listening to them, working in partnership with them and facilitating their choices whenever and wherever possible. Indeed, there is definitely a lack of a correlation between the palliative care and molecular diagnostics in the literature. Therefore, this review may constitute a starting point for extensive research on the importance of molecular diagnostics integration in the care systems and especially for referring patients to the appropriate palliative cares.

\section{Acknowledgment}

We thank all members of the medical biotechnology for their help and support. We are indebted to Mr Hassan Hafidi for his precious support in IT management. We are also grateful to all members of the management team of MAsCIR for their continuous support and finally to the Moroccan government for its financial support.

\section{References}

1. Long MC (1996) Death and dying and recognizing approaching death. Clin Geriatr Med 12(2): 359-368.

2. World Health Organization (WHO) (1990) Cancer pain relief and palliative care. World Health Organization technical report series, Geneva.

3. Quill TE, Lo B, Brock DW (1997) Palliative options of last resort: a comparison of voluntarily stopping eating and drinking, terminal sedation, physician-assisted suicide, and voluntary active euthanasia. JAMA 278(23): 2099-2104.

4. Watson MS, Lucas CF, Hoy AM, Back IN (2005) Oxford handbook of palliative care. ( $1^{\text {st }}$ edn), Oxford University Press.

5. National Council for Hospice and Specialist Palliative Care Services (2002) Definitions of Supportive and Palliative Care. London.

6. The Lewin Group, Inc., (2005) The Value of Diagnostics Innovation, Adoption and Diffusion into Health Care.

7. Cassidy J, Bissett D, Obe RAJS (2002) Oxford Handbook of Oncology. Oxford University Press.

8. Tang YW, Procop GW, Pershing DH (1997) Molecular diagnostics of infectious diseases. Clin Chem 43(11): 2021-2038.

9. Espy MJ, Uhl JR, Sloan LM, Buckwalter SP, Jones MF, et al. (2006) Realtime PCR in clinical microbiology: applications for routine laboratory testing. Clin Microbiol Rev 19(1): 165-256.

10. Gill P, Ghaemi A (2008) Nucleic acid isothermal amplification technologies: a review. Nucleosides Nucleotides Nucleic Acids 27(3): 224-243.

11. Fakruddin M, Mannan KS, Chowdhury A, Mazumdar RM, Hossain MN, et al. (2013) Nucleic acid amplification: Alternative methods of polymerase chain reaction. J Pharm Bioallied Sci 5(4): 245-252.

12. Sepulveda C, Marlin A, Yoshida T, Ullrich A (2002) Palliative Care: 
the World Health Organisation's global perspective. J Pain Symptom Manage 24(2): 91-96.

13. Higginson I (1993) Palliative care: A review of past changes and future trends. J Public Health Med 15(1): 3-8.

14. Scott M, Hall PA (2004) Methods in Molecular Medicine. In Roulston JE \& Bartlett JMS (eds.), Molecular Diagnosis of Cancer. (2ndedn), Humana Press, NJ, USA.

15. World Health Organization (WHO) (2002) National cancer control programmes: policies and managerial guidelines. ( $2^{\text {nd }}$ edn $)$, Geneva.

16. Geels P, Eisenhauer E, Bezjak A, Zee B, Day A (2000) Palliative effect of chemotherapy: objective tumor response is associated with symptom improvement in patients with metastatic breast cancer. J Clin Oncol 18(12): 2395-2405.

17. Ilic D, O'Connor D, Green S, Wilt TJ (2011) Screening for prostate cancer: an updated Cochrane systemic review. BJU Int 107(6): 882891.

18. Dugan JA, Bostwick DG, Myers RP, Qian J, Bergstralh EJ, et al. (1996) The definition and preoperative prediction of clinically insignificant prostate cancer. JAMA 275(4): 288-294.

19. Larkin SE, Holmes S, Cree IA, Walker T, Basketter V, et al. (2012) Identification of markers of prostate cancer progression using candidate gene expression. Br J Cancer 106(1): 157-165.

20. Mazzola CR, Ghoneim T, Shariat SF (2011) Emerging biomarkers for the diagnosis, staging and prognosis of prostate cancer. Prog Urol 21(1): 1-10.
21. Montgomery RB, Mostaghel EA, Vessella R, Hess DL, Kalhorn TF, et al. (2008) Maintenance of intratumoral androgens in metastatic prostate cancer: a mechanism for castration-resistant tumor growth. Cancer Res 68(11): 4447-4454.

22.Goldenberg SL, Bruchovsky N, Gleave ME, Sullivan LD, Akakura $\mathrm{K}$ (1995) Intermittent androgen suppression in the treatment of prostate cancer: a preliminary report. Urology 45(5): 839-844.

23. Storch GA (2000) Diagnostic virology. Clin Infect Dis 31(3): 739-751.

24. Wilson D, Naidoo S, Bekker LG, Cotton M, Maartens G (2003) Handbook of HIV Medicine. Oxford University Press, Oxford, United Kingdom.

25. Bruera E , Higginson IJ, Ripamonti C, Gunten CFV (2006) Textbook of Palliative Medicine. Taylor \& Francis Group, pp. 903-910.

26. Conant MA (1994) The AIDS epidemic. Journal of the American Academy of Dermatology 31(3): S47-S50.

27. Kumar SP, Sisodia V, Ramapuram J (2013) Reporting of HIV/AIDS- A Systematic Review and Quantitative Analysis of Research Publications in Palliative Care Journals. Int J Health Rehabil Sci 2 (1): 56-65.

28. Haynes BF, Pantaleo G, Fauci AS (1996) Towards an understanding of the correlates of protective immunity to HIV infection. Science 271(5247): 324-328.

29.0’Neill J, Selwyn PA, Schietinger H (2003) A Clinical Guide to Supportive and Palliative Care for HIV/AIDS. US Department of Health and Human Services, Washington. 